\title{
VARIABILIDADE TEMPORAL DO ZOOPLÂNCTON NO SISTEMA ESTUARINO DO RIO PARACAUARI (ILHA DO MARAJÓ, PARÁ)
}

\author{
Kelli Garboza da $\operatorname{COSTA}^{1 *}$; Samara Santiago de AZEVEDO²; Luci Cajueiro Carneiro PEREIRA²; \\ Rauquírio Marinho da COSTA2
}

${ }^{1}$ Universidade Federal do Pará, Campus Universitário do Tocantins/Cametá, Travessa Padre Antônio Franco, 2617, Matinha, Cametá-PA, 68.400-000, Brasil. E-mail: garboza@ufpa.br

*Autor correspondente

2 Universidade Federal do Pará, Instituto de Estudos Costeiros, Campus Universitário de Bragança, Alameda Leandro Ribeiro, s/n, Aldeia, Bragança-PA, 68.600-000, Brasil. E-mail: samara santi@hotmail.com, cajueiro@ufpa.br, raucosta@ufpa.br

\begin{abstract}
RESUMO. Coletas nictemerais foram realizadas em uma estação fixa no estuário do rio Paracauari (Pará) para determinar a composição e a densidade do mesozooplâncton (abertura de malha da rede de plâncton de $200 \mu \mathrm{m}$ ) e verificar a influência de alguns fatores ambientais sobre a comunidade zooplanctônica, nos períodos chuvoso e seco. Os valores de temperatura da água $\left(28,5-29,4^{\circ} \mathrm{C}\right)$, salinidade $(0,42-3,14)$, oxigênio dissolvido $\left(4,82-7,8 \mathrm{mg} \cdot \mathrm{L}^{-1}\right)$ e $\mathrm{pH}(7,02-7,29)$ foram mais elevados no período chuvoso. As concentrações de clorofila-a variaram entre $1,59-18,08 \mathrm{mg}^{-3}$. Foram identificados 40 táxons, sendo 17 copépodos e 23 pertencentes a outros táxons. A densidade de copépodos oscilou entre 10,32-243,02 ind. $\mathrm{m}^{-}$ 3 , enquanto que para os outros grupos estes valores variaram entre 2,51-1.039,19 ind. ${ }^{-3}$. Pseudodiaptomus richardi, náuplius de Cirripedia, Tisbe sp., larvas de Gastropoda, outros Calanoida e Pseudodiaptomus gracilis foram os organismos mais representativos em termos de abundância relativa. Os resultados demonstraram que a composição e densidade do mesozooplâncton foram influenciadas pela variação sazonal dos fatores físicos e químicos da água em decorrência, principalmente, da variação na precipitação pluviométrica. Observou-se também uma maior dominância de indivíduos meroplanctônicos nas amostradas coletadas (como larvas de Cirripedia e Gastropoda), o que pode indicar processos reprodutivos de cracas e gastrópodes no estuário do rio Paracauari.
\end{abstract}

Palavras-Chave: meroplâncton, copépodos, variação sazonal, Baía do Marajó, salinidade.

\begin{abstract}
Nyctemeral samples were studied in a fixed station in the Paracauari River estuary (Pará) to determine the composition and density of the mesozooplankton (plankton net $200 \mu \mathrm{m}$ mesh size), and the effect of the environmental factors on the zooplankton community in the rainy and dry seasons. Water temperature $\left(28.5-29.4^{\circ} \mathrm{C}\right)$, salinity $(0.42-3.14)$, dissolved oxygen $\left(4.82-7.8 \mathrm{mg} \cdot \mathrm{L}^{-1}\right.$ in August) and $\mathrm{pH}$ (7.02-7.29) were the highest in the rainy season. Chlorophyll-a concentrations varied between 1.59-18.08 $\mathrm{mg} \cdot \mathrm{m}^{-3}$. A total of 40 taxa were identified, from which 17 belonged to copepods and 23 to other taxonomic groups. Copepods density ranged from 10.32-243.02 ind. $\mathrm{m}^{-3}$, while the other groups showed densities that oscillated from 2.51-1,039.19 ind. $\mathrm{m}^{-3}$. Pseudodiaptomus richardi, Cirripid nauplii, Tisbe sp., Gastropoda larvae, others Calanoida and Pseudodiaptomus gracilis were the more representative taxa in terms of relative abundance. The results showed that the mesozooplankton composition and density were seasonally conditioned to the physical and chemical factors of water, mainly due to rainfall. It was also observed a greater dominance of meroplanktonic individuals in the collected samples (such as Cirripedia and Gastropoda larvae), indicating reproductive processes of barnacles and gastropods in the Paracauari river estuary.
\end{abstract}

Keywords: meroplankton, copepods, seasonal variation, Marajó bay, salinity. 


\section{INTRODUÇÃO}

Em condições naturais, as águas estuarinas são biologicamente mais produtivas do que as do rio e do oceano adjacente, devido às características hidrodinâmicas destes ambientes que, retendo nutrientes, algas e outras plantas, estimulam a elevada produção primária (Elliot and McLusky, 2002). De toda a produção primária realizada por estes ecossistemas, parte desta matéria, sintetizada por meio de processos fotossintéticos, fica armazenada no interior dos estuários e o restante é "exportado" pelas correntes de marés suprindo as necessidades nutricionais dos organismos da região costeira adjacente (Koch and Wolff, 2002).

Os manguezais e a drenagem fluvial são grandes responsáveis pela elevada produtividade observada nos estuários, principalmente na região norte do Brasil, onde se encontra um dos maiores sistemas contínuos de manguezal do mundo (Spalding et al., 1997) e grandes rios, tais como o Amazonas e Pará. A matéria orgânica produzida pelas florestas de mangue e as elevadas descargas dos rios existentes nesta região, constituem uma importante fonte de nutrientes, sustentando uma elevada produção biológica em seus estuários e zonas costeiras adjacentes, favorecendo o desenvolvimento de pequenos mamíferos, aves, peixes, crustáceos e numerosos invertebrados. Os estuários são de grande importância para a biodiversidade e produtividade, pois diversas espécies de interesse comercial utilizam estes ambientes, como áreas de reprodução, desova e desenvolvimento, o que torna importante o conhecimento e a preservação destes ecossistemas.

Dentre os invertebrados presentes nos sistemas estuarinos, destacam-se os organismos zooplanctônicos, que constituem elementos essenciais da fauna aquática, responsáveis pela ciclagem de nutrientes e pelo fluxo de energia entre os produtores primários e os demais consumidores das redes tróficas aquáticas (Carlsson et al., 1995; Takahashi et al., 1984). O zooplâncton constitui uma das comunidades mais abundantes e diversificadas dos ecossistemas aquáticos, contendo inúmeras espécies pertencentes a vários filos, tais como Protozoa, Cnidaria, Ctenophora, Mollusca, Annelida, Arthropoda, Chaetognatha e Chordata (Costa et al., 2011; Costa et al., 2013a; Leite et al., 2009). A predominância de um ou de outro grupo taxonômico desta diversa comunidade depende da combinação de fatores ambientais (temperatura, salinidade, estratificação, poluentes) e biológicos (predação, competição, hábito alimentar) que caracterizam uma determinada região costeira (Adolf et al., 2006). Desta forma, a sucessão ecológica da comunidade zooplanctônica é comum ao longo de um ciclo anual em função da variabilidade ambiental, sendo o predomínio de formas meroplanctônicas, ticoplanctônicas ou holoplanctônicas variável temporalmente.

Existem variações temporais significativas na composição e distribuição da comunidade zooplanctônica na região norte do Brasil (Andrade et al., 2016; Atique et al., 2016; Costa et al., 2008; Costa et al., 2009; Leite et al., 2009; Magalhães et al., 2009). Estas variações temporais estão atribuídas as oscilações no regime pluviométrico, que consequentemente altera os valores 
de salinidade e o input de água fluvial influenciando a comunidade zooplanctônica (Leite et al., 2016; Martorano et al., 1993). Entre os fatores ambientais que influenciam as formas de vida aquáticas, a salinidade regula a estrutura da comunidade planctônica estuarina (Wooldridge, 1999) e a existência de espécies neríticas, principalmente nas desembocaduras dos estuários (Castello, 1985; Montú, 1980).

O conhecimento do zooplâncton, bem como das variáveis físico-químicas da água, são de fundamental importância para estudos de monitoramento ambiental, pois estes organismos respondem rapidamente às modificações ambientais, sendo excelentes bioindicadores de poluição, sazonalidade, desequilíbrio tróficos, entre outros. Portanto, o presente estudo visa determinar a composição da comunidade zooplanctônica no estuário do rio Paracauari (Ilha do Marajó) e avaliar suas relações temporais com parâmetros bióticos (clorofila-a) e abióticos (salinidade, temperatura, $\mathrm{pH}$ e oxigênio dissolvido).

\section{MATERIAL E MÉTODOS \\ ÁREA DE ESTUDO}

O estuário do rio Paracauari (Fig. 1) está localizado na parte leste da Ilha do Marajó

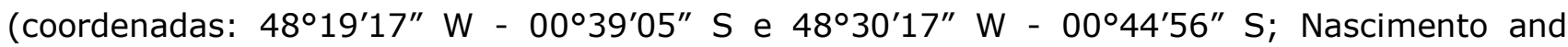
Assunção, 2008), entre os municípios de Soure e Salvaterra, ocupando uma área de $250 \mathrm{~km}^{2}$ (Monteiro, 2009). Como muitos estuários da região, o Paracauari é um sistema estuarino verticalmente homogêneo (Bezerra et al., 2011; Monteiro, 2009), influenciado sazonalmente pelas descargas de sedimentos e água doce do rio Pará. Embora isto represente menos de 5\% do fluxo do rio Amazonas, cerca de $30 \%$ das águas do rio Pará (um dos principais formadores da baía do Marajó), vêm do Amazonas (Callède et al., 2010) através de numerosos canais.

Deste modo, o Paracauari recebe um grande volume de água continental e apresenta um regime semidiurno de macromarés, o que determina a homogeneidade na coluna d'água. A velocidade das correntes atinge $0,70 \mathrm{~m} \cdot \mathrm{s}^{-1}$ em marés de quadratura (Bezerra et al., 2011), podendo atingir velocidades mais elevadas durante as marés de sizígia. Com $51 \mathrm{~km}$ de extensão (Nascimento and Assunção, 2008), o Paracauari está fortemente influenciado pelo regime pluviométrico, o qual apresenta valores anuais de aproximadamente $3.000 \mathrm{~mm}$ (Lima et al., 2005). 


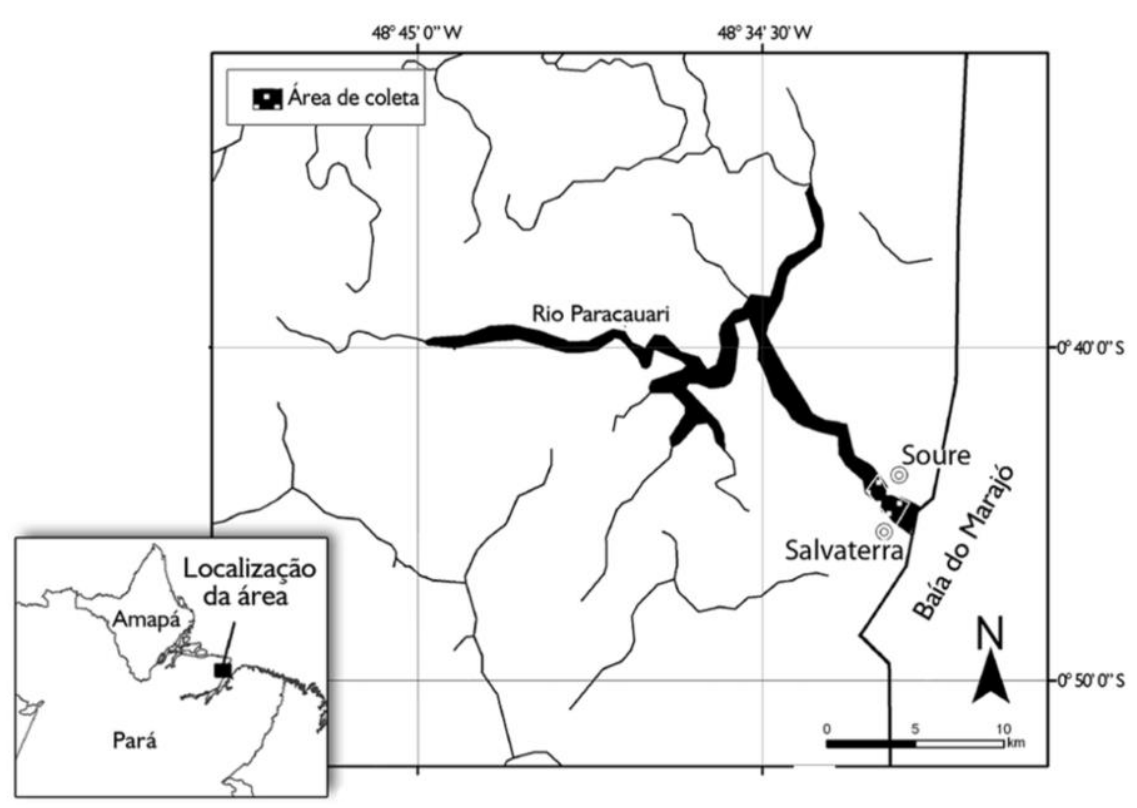

Figura 1. Localização da área de estudo no estuário do rio Paracauari, Ilha do Marajó-PA (modificado de Nascimento and Assunção, 2008).

\section{PROCEDIMENTOS DE CAMPO}

Os valores de temperatura e salinidade foram determinados através de um CTD (modelo XR-420) fixado no píer do IBAMA em Soure (PA), próximo a embarcação fundeada. Amostras de água foram coletadas com garrafa de Niskin $(0-1 \mathrm{~m})$ para determinação dos valores de $\mathrm{pH}$, oxigênio dissolvido e clorofila-a. A fixação do oxigênio dissolvido foi realizada logo após a amostragem de água, utilizando-se $1 \mathrm{~mL}$ de sulfato de manganês e $1 \mathrm{~mL}$ de iodeto alcalino para posterior análises em laboratório. As amostras destinadas as análises de clorofila-a, foram imediatamente acondicionadas em gelo para posterior filtração e análises espectrofotométricas.

A coleta das amostras do material planctônico foi realizada através de arrastos horizontais realizados na subsuperfície da coluna d'água, durante 2-3 minutos, a uma velocidade máxima de 1,5 nós. Para a coleta dos organismos foi utilizada uma rede de plâncton cônico-cilíndrica (50 cm de abertura de boca) com abertura de malha de $200 \mu \mathrm{m}$, acoplada com um fluxômetro mecânico (General Oceanics Inc.) para determinação do volume de água filtrada pela rede.

No final dos arrastos, os organismos coletados foram acondicionados em recipientes plásticos de $500 \mathrm{~mL}$, devidamente etiquetados, contendo formol a $20 \%$ (concentração final de $4 \%$ ), neutralizado com tetraborato de sódio (bórax), segundo a técnica de Newell and Newell (1963). 


\section{ANÁLISES LABORATORIAIS E ESTATÍSTICAS}

A determinação dos valores de $\mathrm{pH}$ foi realizada com auxílio de um pHmetro (Labimeter PH2/PHS-3B). Para a determinação do oxigênio dissolvido foi seguido o método adotado por Winkler, descrito por Strickland and Parsons (1968). As amostras de clorofila-a foram filtradas com auxílio de uma bomba de vácuo com microfiltros de fibra de vidro (Macherey Nagel GF-5, 47 $\mathrm{mm}$ ). A determinação das concentrações de clorofila-a foi realizada através do método espectrofotométrico, descrito por Parsons and Strickland (1963).

Para as análises quali-quantitativas do zooplâncton, as amostras foram fracionadas com o auxílio de um subamostrador de Folsom, segundo a metodologia descrita por Boltovskoy (1981). Os organismos presentes nas subamostras foram identificados e contados, com o auxílio de microscópio estereoscópico (Zeiss) e chaves taxonômicas (Balcer et al., 1984; Björnberg, 1981; Boltovskoy, 1981; Boltovskoy, 1999; Bradford-Grieve et al., 1999; Matsumura-Tundisi, 1986; Trégouboff and Rose, 1957).

Para a análise da estrutura da comunidade foram calculados a densidade zooplanctônica total (ind. $\mathrm{m}^{-3}$ ), abundância relativa, a frequência de ocorrência, a diversidade (Shannon, 1948) e a equitabilidade (Pielou, 1977).

Em relação a análise dos dados foram realizadas análises prévias para determinação da normalidade (teste de Lilliefors) e da homogeneidade (Levene) das variâncias (Sokal and Rohlf, 1969). Quando os dados não apresentaram distribuições normais, transformações log $(x+1)$ foram aplicadas e, posteriormente, a homogeneidade das variâncias foi novamente testada. Para dados homocedásticos foi aplicado teste paramétrico $t$ (Teste-t independente) testando as diferenças existentes entre os meses de coleta e entre os períodos de maré, utilizando-se o nível de significância 0,05. Quando as variâncias foram heterogêneas, o teste não-paramétrico (MannWhitney U) foi aplicado para verificar tais significâncias (Zar, 1999). Todas essas análises foram realizadas com auxílio do programa STATISTICA 6.0.

Para investigar prováveis associações existentes entre as espécies de zooplâncton, os meses, as marés e as amostras coletadas, foi utilizado análise de escalonamento multidimensional não-métrica (NMDS, Non-metric Multi-Dimensional Scaling). Essa análise foi realizada com base em matrizes de similaridade de Bray and Curtis (1957), baseados na densidade das espécies de cada amostra. Os dados de densidade foram transformados em $\log (x+1)$ com a utilização do programa estatístico PRIMER (Plymouth Routines Multivariate Ecological Research), versão 6.1.6 de acordo com Clarke and Warwick (1994). Quando se verificou a formação de grupos no NMDS, a análise de SIMPER foi aplicada para indicar a contribuição percentual das espécies para a similaridade e dissimilaridade dos grupos. O ANOSIM foi aplicado para verificar o grau de similaridade das amostras formados no NMDS e sua significância. Para determinar as relações existentes entre os parâmetros ambientais e (temperatura, salinidade, pH e oxigênio dissolvido) 
e bióticos (densidade, diversidade, equitabilidade e clorofila-a) foram realizadas análises do coeficiente de correlação de Spearman.

\section{RESULTADOS}

Variações significativas nos parâmetros físico-químicos da água foram verificadas apenas para os diferentes períodos sazonais (seco $x$ chuvoso), sendo a salinidade, o pH e o oxigênio dissolvido mais elevados no período seco $(p<0,05)$. As oscilações das variáveis físico-químicas da água do estuário do rio Paracauari durante o estudo podem ser observadas na Tab. 1.

Tabela 1. Variação dos parâmetros físico-químicos da água no estuário do rio Paracauari, Ilha do Marajó (PA).

\begin{tabular}{lccc}
\hline \multicolumn{1}{c}{ Variável } & Mínimo & Máximo & Média \pm DP \\
\hline Temperatura da água $\left({ }^{\circ} \mathrm{C}\right)$ & 28,5 & 29,4 & $29,00 \pm 0,19$ \\
Salinidade & 0,42 & 3,14 & $1,91 \pm 1,21$ \\
$\mathrm{pH}$ & 7,02 & 7,29 & $7,13 \pm 0,09$ \\
Oxigênio dissolvido $\left(\mathrm{mg} \cdot \mathrm{L}^{-1}\right)$ & 4,82 & 7,80 & $6,53 \pm 1,04$ \\
\hline
\end{tabular}

Durante o estudo, foram identificados 40 táxons distribuídos em 9 filos (Sarcomastigophora, Cnidaria, Bryozoa, Annelida, Mollusca, Arthopoda, Echinodermata, Chaetognatha e Chordata), destes táxons, 17 pertencem aos copépodos e 23 a outros grupos taxonômicos. A densidade total do zooplâncton foi de $312,77 \pm 366,95$ ind. $\mathrm{m}^{-3}$, com valores mais elevados no período seco (Teste-t, $\mathrm{p}<0,05$ ), chegando a alcançar 1.282,20 ind. $\mathrm{m}^{-3}$ (Fig. 2B). Esta elevada densidade se deve principalmente a um conjunto de grupos zooplanctônicos (exceto copépodos), que atingiram densidade de 1.039,19 ind.m-3. Padrão oposto foi observado para a biomassa fitoplanctônica (clorofila-a), que apresentou os maiores valores no período chuvoso (Fig. 2A). Os valores de clorofila-a durante o estudo foram de 5,30 $\pm 3,56 \mathrm{mg} \cdot \mathrm{m}^{-3}$ no período seco e 10,84 \pm 4,45 mg.m 3 no período chuvoso.

Durante o período chuvoso (Fig. 3A) os táxons que mais se destacaram, em termos de abundância relativa, foram: Pseudodiaptomus richardi (Dahl F., 1894) (3-50\%), outros Calanoida (0-41\%), Tisbe sp. (4-41\%), larvas de Gastropoda (0-21\%) e Pseudodiaptomus gracilis (F. Dahl, 1894) (0-20\%), enquanto que no período seco (Fig. 3B) destacaram-se os naúplios de Cirripedia (42-93\%), larvas de Gastropoda (0-17\%), P. richardi (1-12\%) e Acartia tonsa (Dana, 1849) (0$8 \%)$.

Durante o período chuvoso foram classificados como muito frequentes, ocorrendo em $100 \%$ das amostras coletadas: P. richardi, Pseudodiaptomus acutus (F. Dahl, 1894), Tisbe sp., Paracalanus quasimodo (Bowman, 1971) e larvas de Gastropoda, seguidos por Leptodiaptomus sicilis (S.A. Forbes, 1882), outros Calanoida e naúplios de Cirripedia com 88\% de ocorrência, e cypris de Cirripedia com 75\% (Fig. 4A). No período seco, os táxons mais frequentes foram: $P$. 
richardi, A. tonsa, larvas de Gastropoda, zoea (Brachyura) e Cirripedia (naúplios e cypris), larvas de peixes, Hidromedusa, P. acutus, P. gracilis, L. sicilis, Leptodiaptomus ashlandi (Marsh, 1893), outros Calanoida e larvas de bivalve com frequência de ocorrência entre 75-100\% (Fig. 4B).

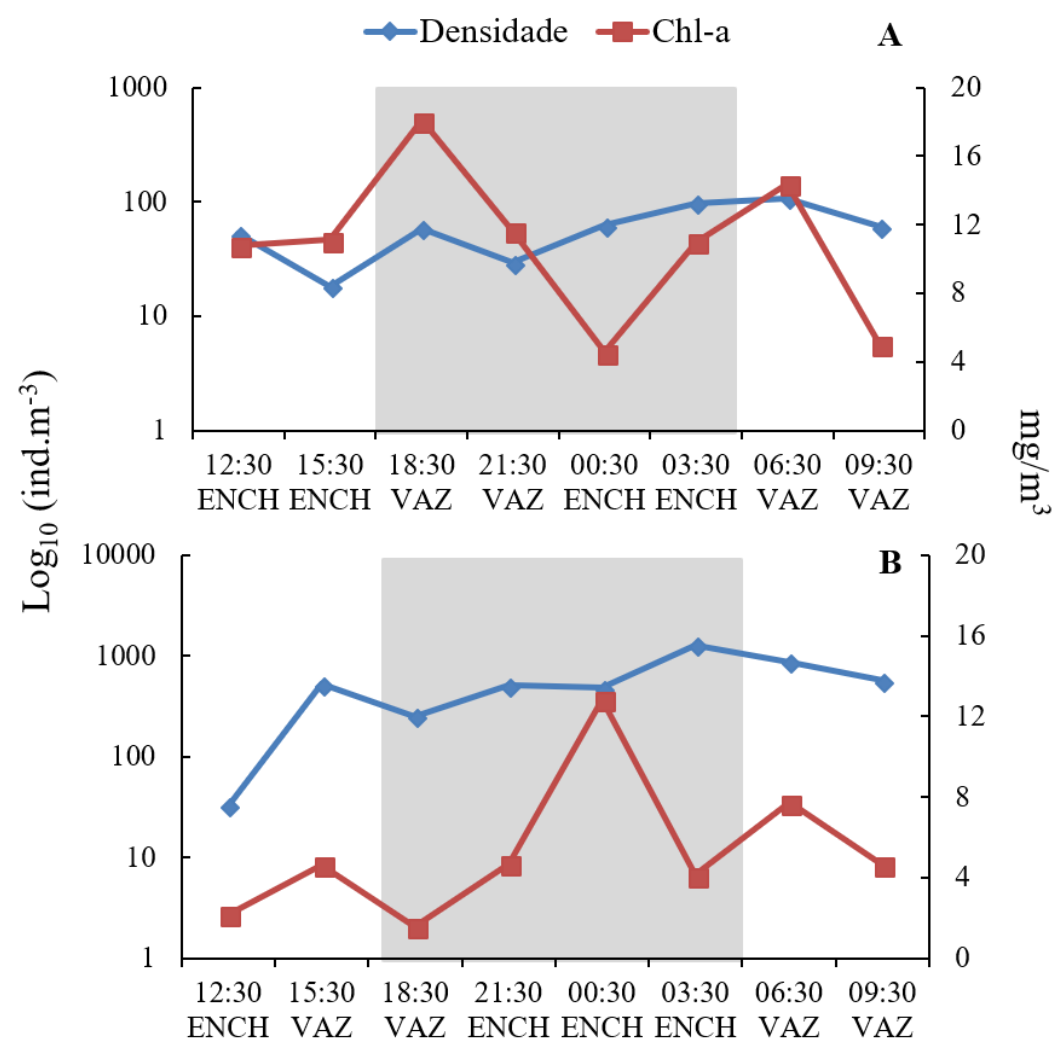

Figura 2. Variação diurna (horário/maré) e sazonal da densidade zooplanctônica total (ind. $\mathrm{m}^{-3}$ ) e da clorofila-a $\left(\mathrm{mg} \cdot \mathrm{m}^{-3}\right)$ no estuário do rio Paracauari, Ilha do Marajó (PA). A - período chuvoso e B - período seco; barra cinza - noite (Notar diferenças nas escalas logarítmicas da densidade).

A diversidade específica $\left(\mathrm{H}^{\prime}\right)$ foi de 2,25 $\pm 0,40$ bits.ind $^{-1}$, com valores oscilando entre 1,35 bits.ind ${ }^{-1}$ (período chuvoso) e 2,97 bits.ind ${ }^{-1}$ (período seco). Embora os valores mais elevados tenham sido registrados no período seco, não houve diferenças significativas entre os períodos sazonais. O mesmo padrão foi observado para a equitabilidade $\left(\mathrm{J}^{\prime}\right)$ que apresentou valor mínimo de 0,52 (período chuvoso) e valor máximo de 0,91 (período seco), com valor médio de 0,76 \pm 0,11 . 
$\square$ Tisbe sp. $\square$ outros Calanoida $\square$ P. richardi $\square$ P. gracilis $\square$ Gastropoda $\mathbf{\square}$ Outros
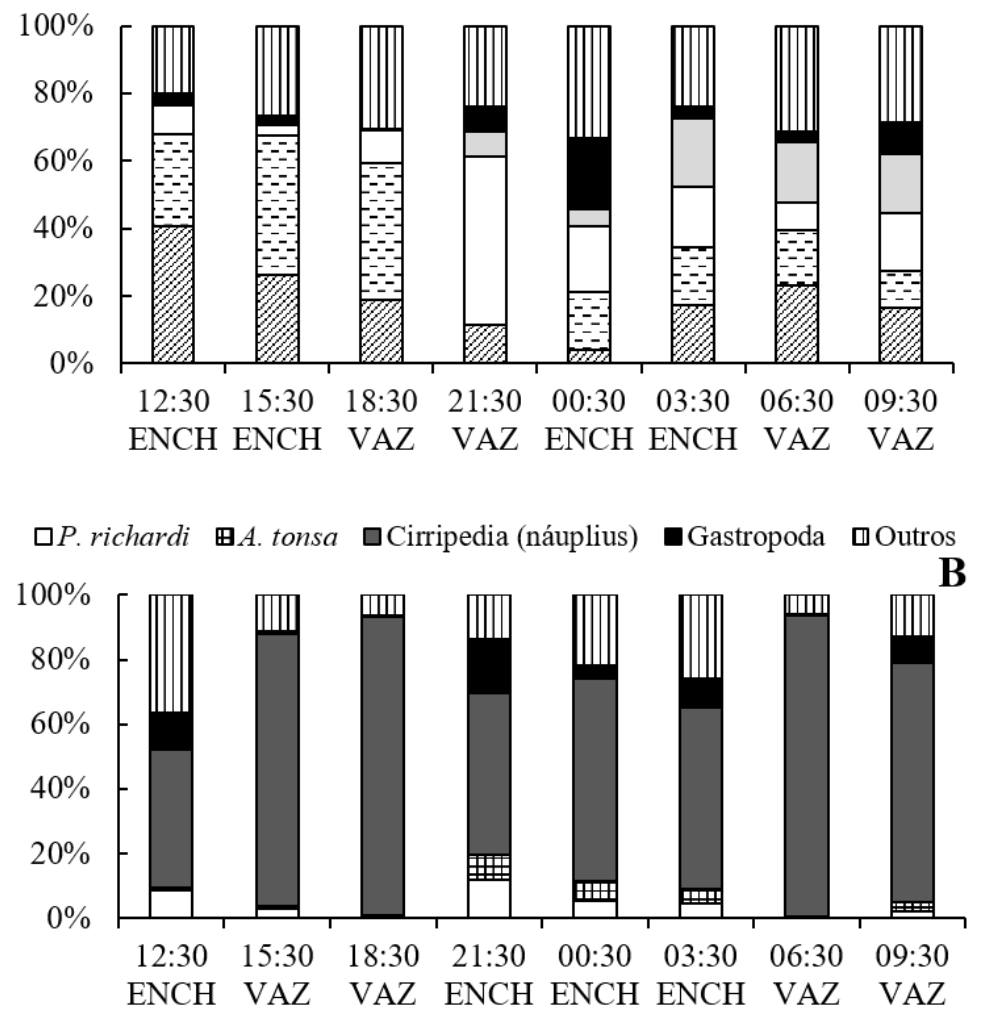

Figura 3. Variações diurna (horário/maré) e sazonal da abundância relativa dos principais táxons no estuário do rio Paracauari, Ilha de Marajó (PA). A - período chuvoso e B - período seco.

$\mathrm{Na}$ análise de escalonamento multidimensional não-métrica (Fig. 5) foi possível verificar a formação de dois grupos distintos: i) o primeiro grupo com similaridade de $56 \%$ entre as amostras do período chuvoso (fevereiro); ii) o segundo grupo com similaridade de $54 \%$ entre as amostras do período seco (agosto). Levando em consideração a formação dos dois grupos é possível confirmar a influência da sazonalidade das chuvas sobre a estrutura da comunidade zooplanctônica (ANOSIM, $p<0,05$ ), bem como a ausência de padrões diário (dia $\mathrm{x}$ noite) e das marés (enchente $x$ vazante), como já observado nas descrições acima. Segundo análise de SIMPER, o grupo chuvoso apresentou maior contribuição percentual de Tisbe sp. $(18,57 \%)$ e o grupo seco de naúplios de Cirripedia $(27,27 \%)$. Como já observado no gráfico de abundância relativa, a contribuição percentual das espécies para cada período sazonal não se restringe apenas a uma única espécie, mas sim a quatro ou mais espécies (ver Fig. 3).

O coeficiente de correlação de Spearman revelou correlações significativas e positivas entre a salinidade e a densidade zooplanctônica (rs: 0,68; $p<0,001$ ), confirmando a elevada concentração de organismos durante o período seco, como já descrito anteriormente. Correlações significativas, porém negativas ( $r s:-0,58 ; p<0,05$ ), foram também registradas entre a 
salinidade e a clorofila-a devido a elevada biomassa fitoplanctônica observada durante o período chuvoso, quando a precipitação aumentou $610 \mathrm{~mm}$ (agosto $=40 \mathrm{~mm}$, fevereiro $=650 \mathrm{~mm}$ ).

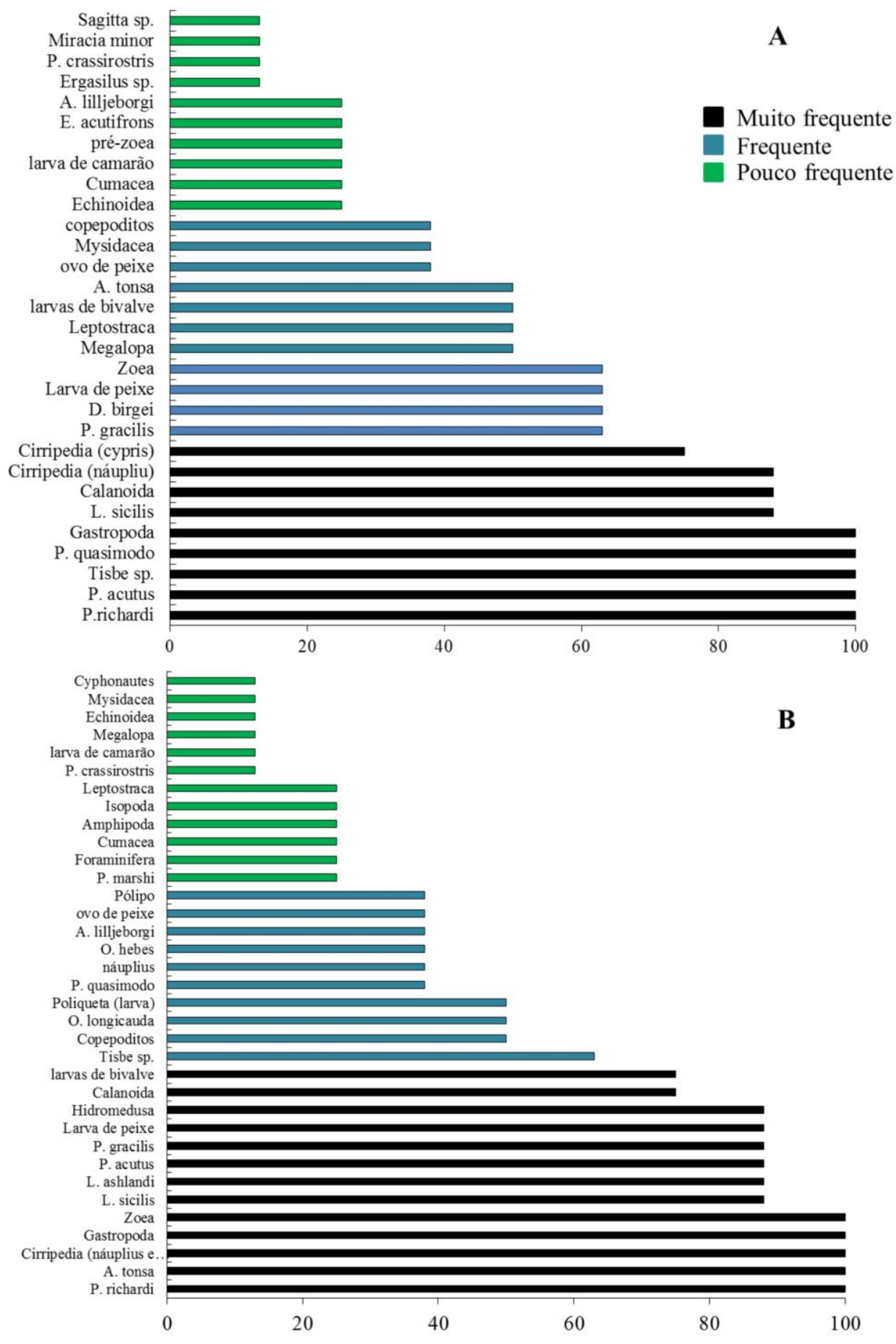

Figura 4. Frequência de ocorrência dos organismos zooplanctônicos identificados nos períodos chuvoso ( $A$ ) e seco (B) de 2009. 


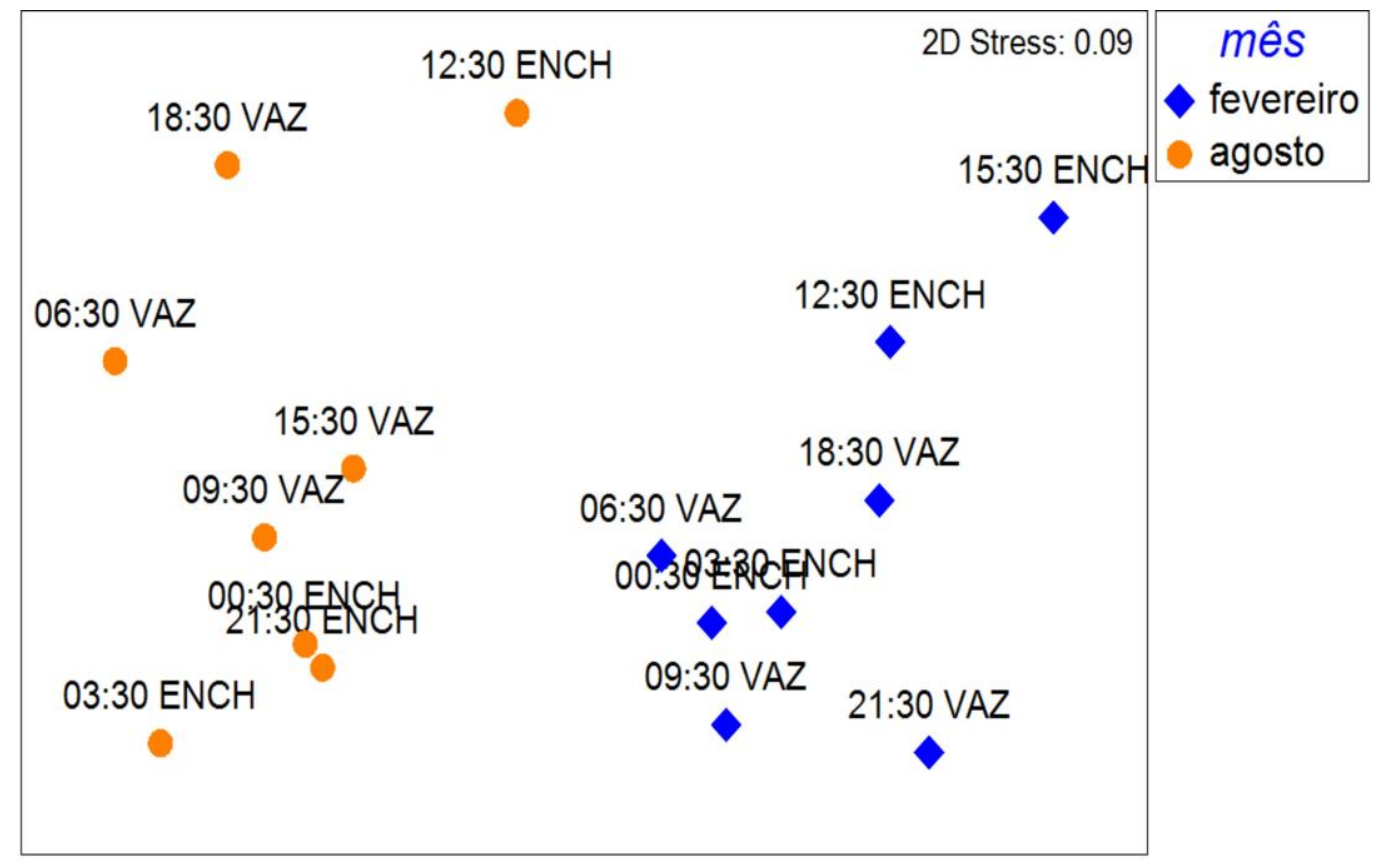

Figura 5. Análise diária (horário/maré) e temporal de escalonamento multidimensional não-métrica (NMDS) no estuário do rio Paracauari, Ilha do Marajó (PA).

\section{DISCUSSÃO}

Como observado em estudos realizados na região amazônica (Costa et al., 2008; Costa et al., 2009; Magalhães et al., 2009; Pereira et al., 2010), as oscilações na temperatura da água não exercem efeito sobre a dinâmica do zooplâncton (Costa et al., 2013a;b). Apesar desta variável afetar o desenvolvimento e a reprodução do zooplâncton estuarino (Froneman, 2001), as flutuações diárias e temporais na temperatura da água no estuário do rio Paracauari foram relativamente discretas, não exercendo efeito sobre a dinâmica temporal do zooplâncton.

No estuário do rio Paracauari, o pH - que pode ser utilizado como indicador de possíveis alterações fisiológicas dos organismos (Cavalcanti et al., 2005) - apresentou uma variação sazonal definida, com menores valores obtidos no período chuvoso em decorrência dos maiores aportes fluviais. Por outro lado, no período seco foram observadas águas mais alcalinas, indicando uma maior influência das águas marinhas. Essas características do $\mathrm{pH}$ são frequentes em ambientes estuarinos devido a dependência do fluxo e refluxo das marés e da quantidade de água doce proveniente dos tributários (Monteiro et al., 2011). O aumento das concentrações de oxigênio dissolvido durante o período seco no estuário do rio Paracauari, pode estar associado à ação dos fortes ventos e correntes que ocorrem neste período (Bezerra et al., 2009), o que provoca uma maior turbulência na superfície da água e intensifica as interações coluna de água/atmosfera.

No litoral amazônico, as elevadas taxas de pluviosidade e o aumento da vazão dos rios são responsáveis por variações temporais na salinidade da água, as quais influenciam a sobrevivência 
e/ou o desenvolvimento dos organismos zooplanctônicos (Costa et al., 2009, Costa et al., 2011, Leite et al., 2016; Magalhães et al., 2009). No Paracauari, os valores de salinidade são inferiores aos obtidos em distintos estuários amazônicos (p. ex. estuário do Taperaçu, estuário do rio Caeté e estuário do rio Curuçá) em decorrência da contribuição de água fluvial e a ausência de ligação direta com o Oceano Atlântico.

Em termos de composição da comunidade zooplanctônica, é comum a dominância de copépodos nos estuários amazônicos (Costa et al., 2009, Costa et al., 2011, Leite et al., 2016), no entanto, o presente estudo aponta como dominante um conjunto de outros grupos taxonômicos, com contribuições sazonais pontuais de organismos meroplanctônicos, como cirripédios e gastrópodes. Segundo Hirst et al. (1999) e Muxagata et al. (2004) espécies de Cirripedia podem contribuir tanto quanto os copépodos para a produção secundária em ambientes costeiros, o que demonstra a importância destes organismos para a produtividade local.

Durante o presente estudo foi observada uma possível influência da densidade zooplanctônica sobre a concentração de clorofila-a, ambas mostrando picos inversos ao longo dos meses estudados. O efeito do início da estação chuvosa sobre a biomassa fitoplanctônica possivelmente está associado a maior hidrodinâmica e descarga fluvial e, consequentemente, ressuspensão dos nutrientes inorgânicos na coluna d'água. Por outro lado, geralmente, a variação na biomassa fitoplanctônica pode estar diretamente relacionada ao grazing pelo zooplâncton (Albaina et al., 2009; Grange et al., 2000) o que poderia explicar os picos inversos observados durante o estudo.

Diversos autores têm relatado a dominância do meroplâncton nos sistemas aquáticos relacionado a altas concentrações de biomassa e produtividade, ou aos padrões reprodutivos de algumas espécies bentônicas (Costa et al., 2013a; Jamet et al., 2001; Leite et al., 2009). Em adição a dominância dos organismos meroplanctônicos (como cirripédios), altas densidades de copépodos foram registradas durante o presente estudo, padrão este observado também em outros estuários (Champalbert et al., 2007; Leite et al., 2009; Magalhães et al., 2009).

Algumas espécies são adaptadas fisiologicamente as alterações da salinidade, como por exemplo, o copépodo Pseudodiaptomus richardi que ocorreu durante todo o estudo, como também observado por Costa et al. (2013b). Apesar de $P$. richardi ter características eurihalinas (Montú, 1980), estudos realizados na região amazônica enfatizam a correlação negativa existente entre a densidade de $P$. richardi e a salinidade (Krumme and Liang, 2004; Magalhães et al., 2006), demonstrando assim a preferência de organismos desta espécie por águas menos salinas.

No estuário do rio Paracauari, o aumento dos índices de diversidade e equitabilidade nas amostras coletadas no período seco indica a influência de água marinha, a qual recruta espécies costeiras dos ecossistemas aquáticos adjacentes, como observado em outros ecossistemas estuarinos no Brasil (Costa et al., 2011) e no mundo (Froneman, 2004; Li et al., 2006). 
A estrutura da comunidade zooplanctônica no estuário do rio Paracauari, por meio das análises de similaridade, demonstrou uma separação nítida das amostras nos diferentes períodos sazonais. Esta separação foi ocasionada principalmente pelas variações no regime pluviométrico local, que por sua vez influenciaram os valores de salinidade, como previamente descrito para diversos sistemas costeiros da região norte (Atique et al., 2016; Costa et al., 2008, Costa et al., 2013a, entre outros).

De forma geral, o estuário do rio Paracauari não apresenta variações diurnas, visto que a homogeneidade na coluna d'água, decorrente da forte hidrodinâmica local, "limita" a migração vertical dos organismos zooplanctônicos. Por outro lado, a pluviometria constitui o principal fator responsável pela variação sazonal da salinidade, que por sua vez influencia diretamente a estrutura da comunidade zooplanctônica, a qual apresenta, no estuário do Paracauari, a dominância de organismos holoplanctônicos durante o período chuvoso e meroplanctônicos durante o período de estiagem. As descargas fluviais decorrentes no estuário do rio Paracauari, a pluviometria local e a lavagem das planícies de inundação (manguezais) favorecem o desenvolvimento da biomassa fitoplanctônica, propiciando o crescimento da comunidade zooplanctônica, a qual apresentou elevadas densidades, principalmente, no período seco quando os valores de clorofila-a reduziram.

\section{AGRADECIMENTOS}

A FAPESPA pelo financiamento dos projetos Universal (115/2008) e PROSGRAD (070/2008), os quais o presente estudo esteve vinculado. Os autores agradecem ao CNPq pela concessão da bolsa de mestrado à primeira autora, e pelo suporte financeiro oferecido através dos projetos CTHidro casadinho (\# 552126/2005-5) e Taxonomia (\# 563967/05-6), bem como pelas bolsas de pesquisa \# 308379/2011-0 e \# 306061/2011-2, concedidas ao Dr. Rauquírio Marinho da Costa e a Dra. Luci Cajueiro Carneiro Pereira, respectivamente.

\section{REFERÊNCIAS}

Adolf, J.E., Yeager, C.L., Miller, W.D., Mallonee, M.E. and Harding, L.W. (2006), Environmental forcing of phytoplankton floral composition, biomass, and primary productivity in Chesapeake Bay, USA, Estuarine, Coastal and Shelf Science, Vol. 67, pp. 108-22.

Albaina, A., Villate, E. and Uriarte, I. (2009), Zooplankton communities in two contrasting Basque estuaries (1999-2001): reporting changes associated with ecosystem health, Journal of Plankton Research, Vol. 31, pp. 739-52.

Andrade, M.P., Magalhães, A., Pereira, L.C.C., Flores-Montes, M., Pardal, E.C., Andrade, T.P. and Costa, R.M. (2016), Effects of a La Niña event on hydrological patterns and copepod 
community structure in a shallow tropical estuary (Taperaçu, Northern Brazil), Journal of Marine Systems, Vol. 164, pp. 128-43.

Atique, P., Costa, K.G., Monteiro, M.C., Pereira, L.C.C. and Costa, R.M. (2016), Copepod assemblages in a highly dynamics equatorial estuary on the Brazilian Amazon Coast, Marine Ecology, Vol. 1, pp. 1-14.

Balcer, M.D., Korda, N.L. and Dodson, S.I. (1984), Zooplankton of the Great Lakes: A Guide to the Identification and Ecology of the Common Crustacean Species, University of Wisconsin Press, London.

Bezerra, M.O., Akel, A.R., Araújo, T.R.C., Krelling, A.P.M. and Redondo, J.M. (2009), Application of aerial video images for lagrangian tracer studies in maritime and estuarine port zones, Journal of Coastal Research, Special issue 56, pp. 787-91.

Bezerra, M.O., Medeiros, C., Krelling, A.P.M., Rosário, R.P. and Rollnic, M. (2011), Physical oceanographic behavior at the Guama/Acara-Moju and the Paracauari river mouths, Amazon Coast (Brazil), Journal of Coastal Research, Special issue 64, pp.1448-52.

Björnberg, T.K.S. (1981), Copepoda, in Boltovskoy, D. (Ed.), Atlas del Zooplancton del Atlántico Sudoccidental y métodos de trabajo con el zooplancton marino, Instituto Nacional de Investigación y Desarrollo Pesquero-INIDEP, Mar del Plata, pp. 587-679.

Boltovskoy, D. (1981), Atlas del Zooplancton del Atlántico Sudoccidental y métodos de trabajo con el zooplancton marino, Instituto Nacional de Investigación y Desarrollo PesqueroINIDEP, Mar del Plata.

Boltovskoy, D. (1999), South Atlantic Zooplankton, Backhuys, Leiden.

Bradford-Grieve, J.M., Markhaseva, E.L., Rocha, C.E.F., Abiahy, B. (1999), Copepoda, in Boltovskoy, D. (Ed.), South Atlantic Zooplankton, Bachyuys, Netherlands, pp. 869-1098.

Bray, J.R. and Curtis, J.T. (1957), An ordination of the upland forest communities of southern Wisconsin, Ecological Monographies, Vol. 27, pp. 325-49.

Callède, J., Cochonneau, G., Ronchail, J., Vieira Alvez, F., Guyot, J.-L., Santos Guimarues, V. and De Oliveira, E. (2010), Les apports en eau de I'Amazone a I'ocean Atlantique, Revue des sciences de l'eau/Journal of Water Science, Vol. 23(3), pp. 247-73.

Carlsson, P., Granéli, E., Finenko, G. and Maestrini, S. Y. (1995), Copepod grazing on a phytoplankton community containing the toxic dinoflagellate Dinophysis acuminate, Journal of Plankton Research, Vol. 17, pp. 1925-38.

Castello, J.P. (1985), La ecologia de los consumidores del estuario de Lagoa dos Patos, Brasil, in Yanez-Aranciabia, A. (Ed.), Fish community ecology in estuaries and coastal lagoons: Towards an Ecosystem Integration, UNAM Press, México, pp. 383-406. 
Cavalcanti, L.B., Macedo, S.J. and Montes, M.J.F. (2005), Condições hidrológicas associadas ao cultivo do camarão marinho Litopenaeus vannamei na região estuarina do Rio Paraíba do Norte (Paraíba Brasil), Revista da Associação Brasileira de Criadores de Camarão, Vol. 7, pp. 60-65.

Champalbert, G., Pagano, M., Sene, P. and Corbin, D. (2007), Relationships between meso- and macro-zooplankton communities and hydrology in the Senegal River Estuary, Estuarine, Coastal and Shelf Science, Vol. 75, pp. 381-94.

Clarke, K.R. and Warwick, R.M. (1994), Change in marine communities: an approach to statistical analysis and interpretation, Plymouth Marine Laboratory, Plymouth.

Costa, K.G., Pereira, L.C.C. and Costa, R.M. (2008), Short and long-term temporal variation of the zooplankton in a tropical estuary (Amazon region, Brazil), Boletim do Museu Paraense Emílio Goeldi, Vol. 3, pp. 127-41.

Costa, K.G., Bezerra, T.R., Monteiro, M.C., Vallinoto, M., Berrêdo, J.F., Pereira, L.C.C. and Costa, R.M. (2013a), Tidal-Induced Changes in the Zooplankton Community of an Amazon Estuary, Journal of Coastal Research, Vol. 29, pp. 756-65.

Costa, K.G., Bessa, R.S.C., Pereira, L.C.C. and Costa, R.M. (2013b), Short and medium-term changes of Pseudodiaptomidae copepods in the Amazonian Mangrove Coast: the Paracauari River estuary, Journal of Coastal Research, Special issue 65, pp. 1116-21.

Costa, R.M., Leite, N.R. and Pereira, L.C.C. (2009), Mesozooplankton of the Curuçá Estuary (Amazon Coast, Brazil), Journal of Coastal Research, Special issue 56, pp. 400-04.

Costa, R.M., Atique, P., Costa, K.G. and Pereira, L.C.C. (2011), Seasonal and spatial variation in hydrological parameters and microzooplankton communities in an Amazonian estuary, Journal of Coastal Research, Special issue 64, pp.1477-81.

Elliot, M. and McLusky, D.S. (2002), The need for definitions in understanding estuaries, Estuarine, Coastal and Shelf Science, Vol. 55, pp. 815-27.

Froneman, P.W. (2001), Seasonal changes in zooplankton biomass and grazing in a temperature estuary, South Africa, Estuarine, Coastal and Shelf Science, Vol. 52, pp. 543-53.

Froneman, P.W. (2004), Zooplankton community structure and biomass in a southern African temporarily open/closed estuary, Estuarine, Coastal and Shelf Science, Vol. 60, pp. 12532.

Grange, N., Whitfield, A.K., De Villiers, C.J. and Allanson, B.R. (2000), The response of two South African east coast estuaries to altered river flow regimes, Aquatic Conservation: Marine and Freshwater Ecosystems, Vol. 10, pp. 155-77. 
Hirst, A.G., Sheader, M. and Williams, J.A. (1999), Annual pattern of calanoid copepod abundance, prosome length and minor role in pelagic carbon flux in the Solent, UK, Marine Ecology Progress Series, Vol. 177, pp. 133-46.

Jamet, J.L., Boge, G. and Richard, S. (2001), Zooplankton communities in bays of Toulan area (Northwest Mediterranean Sea, France), Hydrobiologia, Vol. 457, pp. 155-65.

Koch, V. and Wolff, M. (2002), Energy budget and ecological role of mangrove epibenthos in the Ceté estuary, North Brazil, Marine Ecology Progress Series, Vol. 228, pp. 119-30.

Krumme, U. and Liang, T. (2004), Tidal-induced changes in a copepod-dominated zooplankton community in a macrotidal mangrove channel in Northern Brazil, Zoological Studies, Vol. 43, p. $404-14$.

Leite, N.R., Pereira, L.C.C. and Costa, R.M. (2009), Distribuição temporal do mesozoplâncton no furo Muriá, Pará, Brasil, Boletim do Museu Paraense Emílio Goeldi, Vol. 4, pp. 149-164.

Leite, N.R., Magalhães, A., Silva, L.M., Andrade, T.P., Matos, J.B., Costa, A.K., Pereira, L.C.C., Flores-Montes, M.J. and Costa, R.M. (2016), Short and Medium Term Variation in the Dynamics of the Mesozooplankton Community of an Amazonian Estuary, Journal of Coastal Research, Special issue 75, pp. 218-22.

Li, K.Z., Yin, J.Q., Huang, L.M. and Tan, Y.H. (2006), Spatial and temporal variations of mesozooplankton in the Pearl River estuary, China, Estuarine, Coastal and Shelf Science, Vol. 67, pp. 543-52.

Lima, A.M.M., Oliveira, L.L., Fontinhas, R.L. and Lima, R.J.S. (2005), Ilha do Marajó: Revisão histórica, hidroclimatologia, bacias hidrográficas e propostas de gestão, Holos Environment, Vol. 5, pp. 65-80.

Magalhães, A., Costa, R.M., Liang, T.-H., Pereira, L.C.C. and Ribeiro, M.J.S. (2006), Spatial and temporal distribution in density and biomass of two Pseudodiaptomus species (Copepoda: Calanoida) in the Caeté river estuary (Amazon region - North of Brazil), Brazilian Journal of Biology, Vol. 66, pp. 421-30.

Magalhães, A., Leite, N.R., Silva, J.G.S., Pereira, L.C.C. and Costa, R.M. (2009), Seasonal variation in the copepod community structure from a tropical Amazon estuary, Northern Brazil, Anais da Academia Brasileira de Ciências, Vol. 81, pp. 187-97.

Martorano, L.G., Pereira, L.C., Cézar, E.G.M. and Pereira, I.C.B. (1993), Estudos Climáticos do estado do Pará, Classificação Climática (Koppen) e Deficiência Hídrica (Thornthwhite, Mather), SUDAM/EMBRAPA, SNLCS, Belém.

Matsumura-Tundisi, T. (1986), Latitudinal distribution of Calanoida copepods in freshwater aquatic system of Brazil, Revista Brasileira de Biologia, Vol. 46, pp. 527-53. 
Monteiro, M.C., Pereira, L.C.C. and Oliveira, S.M.O. (2009), Morphodynamic changes of a macrotidal sand beach in the Brazilian Amazon coast (Ajuruteua-Pará), Journal of Coastal Research, Special issue 56, pp. 103-07.

Monteiro, M.C., Pereira, L.C.C., Guimarães, D.O., Costa, R.M., Souza-Filho, P.W.M., Vieira, S.R. and Jiménez, J.A. (2011), Influence of natural and anthropogenic conditions on the water quality of the Caeté river estuary (North Brazil), Journal of Coastal Research, Special issue 64, pp. 1535-39.

Montú, M. (1980), Zooplâncton do estuário da Lagoa dos Patos. I. Estrutura e variações temporais e espaciais da comunidade, Atlântica, Vol. 4, pp. 53-72.

Muxagata, E., William, J.A. and Sheader, M. (2004), Composition and temporal distribution of cirripede larvae in Southampton Water, England, with particular reference to the secondary production of Elminius modestus, ICES Journal of Marine Science, Vol. 61, pp. 585-95.

Nascimento, F.L. and Assunção, M.I.S. (2008), Ecologia reprodutiva dos tralhotos Anableps anableps e Anableps microlepis (Pisces: Osteichthyes: Cyprinodontiformes: Anablepidae) no rio Paracauari, ilha de Marajó, Pará, Brasil, Boletim do Museu Paraense Emílio Goeldi, Vol. 3, pp. 229-40.

Newell, G.H. and Newell, R. (1963), Marine plankton: a pratical guide, Edutat, Hutchinson.

Parsons, T.R. and Strickland, J.D.H. (1963), Discussion of spectrophotometric determination of marine plankton pigments, with revised equations of certaining chlorophyll $a$ and carotenoids, Journal of Marine Research, Vol. 21, pp. 155-163.

Pereira, L.C.C., Monteiro, M.C., Guimarães, D.O., Matos, J.B. and Costa, R.M. (2010), Seasonal effects of wasterwater to the water quality of the Caeté river estuary, Brazilian Amazon, Anais da Academia Brasileira de Ciências, Vol. 82, pp. 467-78.

Pielou, E.C. (1977), Mathematical ecology, John Wiley and Sons, New York.

Shannon, C.E. (1948), A mathematical theory of communication, Bell System Technical Journal, Vol. 27, pp. 379-423.

Sokal, R.R. and Rohlf, F.J. (1969), Biometry - The principles and practice of numerical classification in biological research, W. H. Freeman, San Francisco.

Spalding, M., Blasco, F. an Field, C.D. (1997), World Mangrove Atlas, The International Society for Mangrove Ecosystems, Okinawa.

Strickland, J.D.H. and Parsons, T.R. (1968), A practical handbook of seawater analysis (2nd ed.), Bulletin of Fisheries Board of Canada, Vol. 167, pp. 1-311. 
Takahashi, M., Hargrave, B. and Parsons, T. (1984), Biological Oceanographic Processes (3rd ed.), Pergamon International Library of Science, Technology, Engineering and Social Studies, New York.

Trégouboff, G. and Rose, M. (1957), Manuel de planctonologie mediterranéenne, Centre Nacional de la Recherche Scientifique, Paris.

Wooldridge, T.H. (1999), Estuarine zooplankton community structure and dynamics, in Allanson, B.R. and Baird, D. (Eds.), Estuaries of South Africa, Cambridge University Press, Cambridge, pp. 141-66.

Zar, J.H. (1999), Bioestatistical analysis, Prentice Hall, New Jersey. 